\title{
Efficacy of Low-Level Laser Therapy on Hand Foot Syndrome Associated with Chemotherapy
}

\author{
Hend Ali Mahmoud Ibrahim Badr al-din*1, Amal Mohamed Abd El baky',
} Mohammed Abdel Rahman'2, Karim Ibrahim Saafan ${ }^{1}$

${ }^{1}$ Departments of Physical Therapy for Surgery - Faculty of Physical Therapy, Cairo University, ${ }^{2}$ Department of

Oncology - Faculty of Medicine, Cairo University, Cairo University

*Corresponding author: Hend Ali Mahmoud Ibrahim Badr Al-Din, Mobile: (+20) 01117047800,

E-Mail: dr.hend.reda@gmail.com

\begin{abstract}
Background: we speculate that low level laser might decrease the inflammatory reactions and pain associated with hand foot syndrome because low-level laser therapy, has proven high efficacy in the management of chemotherapy/radiation-induced oral mucositis and radiation-induced dermatitis that might share some physiopathological characteristics with hand foot syndrome,

Objective: To determine whether low-level laser therapy (LLLT) might be effective for chemotherapy-induced hand foot syndrome (HFS).

Patients and methods: This study included thirty patients with hand foot syndrome (grade I -II - III according to WHO definition) after treatment with chemotherapy from both sexes. Their ages ranged from 35-60 years. Patients were randomly assigned into two equal groups in number. Group A (the study group) included fifteen patients received low-level laser therapy in addition to the traditional medical treatment. Group B (control group) included fifteen patients received the traditional medical treatment. The participants of both groups were assessed by the following tools to assess the impact of HFS on quality of life by visual analogue scale and HFS-14 questionnaire.

Results: There was a significant decrease in VAS and HFS-14 questionnaire score post treatment in the study and control groups compared to that of pretreatment $(\mathrm{p}<0.001)$. The percentages of decrease in VAS and HFS-14 questionnaire score in the study group were $48.29 \%$ and $34.22 \%$ respectively, while for the control group, they were $28.75 \%$ and $18.82 \%$ respectively.
\end{abstract}

Conclusion: Based on this study, it could be concluded that LLLT might represent a useful approach for the management of HFS.

Keywords: Hand foot syndrome, Low-level laser, Chemotherapy-induced toxicity.

\section{INTRODUCTION}

Hand-foot syndrome (also known as hand-foot reaction, palmar-plantar erythrodysesthesia, chemotherapy-associated acral erythema or Burgdorf reaction) is a cutaneous toxicity that is most frequently linked to cytotoxic drugs (e.g., 5-fluorouracil, capecitabine, cytarabine, docetaxel and pegylated liposomal doxorubicin) and multikinase-inhibitors ${ }^{(\mathbf{1})}$. It usually occurs 2-12 days after administration of chemotherapy. The initial symptoms are palmoplantar dysesthesia and tingling, which can evolve within a few days to burning pain and symmetric well-defined erythema with edematous swelling ${ }^{(2)}$. In severe cases, blistering, desquamation and subsequent ulceration can occur. It usually resolves within a few weeks after withdrawal of the responsible drug ${ }^{(3)}$.

Histologic features of HFS are non-specific and based on a small number of patients. They include vacuolar degeneration of the basal cell layer, mild spongiosis, keratinocytes necrosis, papillary dermal edema, lymph histiocytic infiltrates and partial separation of the epidermis from the dermis. Perivascular infiltrates composed of lymphocytes and eosinophils are often seen in the dermis. There may also be evidence of eccrine squamous syringe metaplasia or neutrophilic eccrine hidradenitis ${ }^{(4)}$.
Low-level laser or light therapy (LLLT) has shown significant promise. LLLT refers to light therapy that may stimulate tissue regeneration, reduce inflammation and control pain ${ }^{(5)}$. LLLT mediated vascular regulation, increases tissue oxygenation and allows for greater traffic of immune cells, which may contribute to the promotion of wound repair and regeneration. Moreover, LLLT when delivered appropriately reduces pain and improves function ${ }^{(6)}$.

The present study was designed to investigate the effect of low-level laser therapy on the management of the hand-foot syndrome associated with cancer therapy.

\section{MATERIALS AND METHODS}

Thirty patients who had HFS participated in this study. Their ages ranged from 35 to 60 years. The participants were selected from Oncology Teaching Hospital in Ismailia. Patients were randomly assigned into two equal groups in number. Group A (the study group) included fifteen patients that received lowlevel laser therapy in addition to the traditional medical treatment and group B (control group) that included fifteen patients who received the traditional medical treatment. Assessment of the impact of handfoot syndrome (HFS) on quality of life in both groups 
was conducted by visual analogue scale and HFS_14 questionnaire.

\section{Ethical approval:}

This study was approved by the Research Ethics Committee of Physical Therapy College, Cairo University. Every patient signed an informed written consent for acceptance of the operation.

\section{Inclusion criteria:}

All patients were pathologically proven cancer and received drugs that known to cause hand foot syndrome e.g., fluorouracil infusion and oral capecitabine, and diagnosed with hand foot syndrome by oncologist. Their ages ranged from 35-60 years old.

\section{Exclusion criteria:}

The patient who had metallic implants at or near hand and foot and who had cardiac pacemakers or other implanted electronic devices. Also, patients who had any significant health problem such as circulatory disorders or history of skin malignancy in the area to be treated as well as patients who were uncooperative and patients who missed more than 3 sessions.

\section{Instrumentation:}

The study instrumentation were divided into measurement and therapeutic equipment:

\section{Measurement tools:}

- Visual analogue scale (VAS): Which consisting of a $10-\mathrm{cm}$ horizontal line. The origin of the line on the left side is designated "no pain" and the end of the line on the right side is designated "worst pain ever ${ }^{(7)}$.

- Hand foot syndrome-14 questionnaire: A specific quality of life (QOL) scale developed for patients suffering from hand-foot syndrome. This scale specifically developed for patients with HFS is a valid and valuable tool for measuring HFS-related quality of life impairment with a maximum score of 100 and a minimum score of 2. A higher score indicates poorer QOL ${ }^{(8)}$.

\section{Therapeutic equipment:}

This was scanner laser therapy unit: M6 therapy laser unit with frequency of $500 \mathrm{~Hz}$, power level of $50 \%$ and energy of $5.24 \mathrm{~J} / \mathrm{cm}^{2}$ was applied to the whole surface of the affected palms or soles or both 3 times a week in addition to the traditional medical treatment. The patients were asked to evaluate quality of life impairment using HFS-14 questionnaire pre and post treatment period (1 month).

\section{Statistical analysis}

Descriptive statistics and unpaired t-test were conducted for comparison of age between groups. Chi-squared was carried out for comparison of sex distribution between groups. Normal distribution of data was checked using the Shapiro-Wilk test for all variables. Levene's test for homogeneity of variances was conducted to test the homogeneity between groups. Unpaired t-test was conducted to compare the mean values of VAS and HFS-14 questionnaire between the study and control groups.

Paired t-test was conducted for comparison between pre- and post-treatment in each group. The level of significance for all statistical tests was set at $p$ $\leq 0.05$. All statistical analysis was conducted through the statistical package for social studies (SPSS) version 25 for windows (IBM SPSS, Chicago, IL, USA).

\section{RESULTS}

Table (1) showed the subject characteristics of the study and control groups. There was no significant difference between both groups concerning age and sex distribution $(\mathrm{p}>0.05)$.

Table (1): Basic characteristics of participants

\begin{tabular}{|lccc|}
\hline & $\begin{array}{c}\text { Study } \\
\text { group }\end{array}$ & $\begin{array}{c}\text { Control } \\
\text { group }\end{array}$ & $\begin{array}{c}\text { p- } \\
\text { value }\end{array}$ \\
\cline { 2 - 3 } & $\begin{array}{c}\text { Mean } \pm \\
\text { SD }\end{array}$ & $\begin{array}{c}\text { Mean } \pm \\
\text { SD }\end{array}$ & \\
\cline { 2 - 3 } Age (years) & $47.2 \pm$ & $49.13 \pm$ & 0.53 \\
Sex, n (\%) & 7.47 & 9.14 & \\
Females & & & \\
Males & $6(40 \%)$ & $7(47 \%)$ & 0.71 \\
\hline SD, standard deviation. & $9(60 \%)$ & $8(53 \%)$ & \\
\hline
\end{tabular}

significance.

Effect of treatment on VAS and HFS-14 questionnaire:

\section{Comparison within group:}

There was a significant decrease in VAS and HFS-14 questionnaire score post-treatment in the study and control groups compared to that of pretreatment $(p<0.001)$. The percent of decrease in VAS and HFS-14 questionnaire score in the study group was $48.29 \%$ and $34.22 \%$ respectively, while that for control group was $28.75 \%$ and $18.82 \%$ respectively (Table 2).

\section{Comparison between groups:}

There was no significant difference between both groups pre-treatment $(p>0.05)$. Comparison between both groups post-treatment revealed a significant decrease in VAS and HFS-14 questionnaire score of the study group compared to that of the control group $(\mathrm{p}<0.05)$ as shown ion table 2 .

Table (2): Mean VAS and HFS-14 questionnaire pre- and post-treatment of the study and control groups

Study Control group




\begin{tabular}{|c|c|c|c|c|c|}
\hline & group & & & & \\
\hline & Mean \pm SD & Mean \pm SD & MD & t- value & p value \\
\hline VAS & & & & & \\
\hline Pre treatment & $7.6 \pm 1.18$ & $7.2 \pm 1.26$ & 0.4 & 0.89 & 0.37 \\
\hline Post treatment & $3.93 \pm 1.03$ & $5.13 \pm 1.45$ & -1.2 & -2.6 & 0.01 \\
\hline MD & 3.67 & 2.07 & & & \\
\hline$\%$ of change & 48.29 & 28.75 & & & \\
\hline t- value & 17.39 & 6.88 & & & \\
\hline & $P=0.001$ & $P=0.001$ & & & \\
\hline HFS-14 questio & & & & & \\
\hline Pre treatment & $50.06 \pm 9.61$ & $48.2 \pm 10.5$ & 1.86 & 0.5 & 0.61 \\
\hline Post treatment & $32.93 \pm 5.98$ & $39.13 \pm 7.97$ & -6.2 & -2.4 & 0.02 \\
\hline MD & 17.13 & 9.07 & & & \\
\hline$\%$ of change & 34.22 & 18.82 & & & \\
\hline t- value & 12.41 & 7.42 & & & \\
\hline & $P=0.001$ & $P=0.001$ & & & \\
\hline
\end{tabular}

$\mathrm{SD}$, standard deviation; $\mathrm{MD}$, mean difference; $\mathrm{p}$-value, probability value.

\section{DISCUSSION}

The present study was designed to investigate the effect of low-level laser therapy on the management of the hand-foot syndrome associated with cancer therapy. This study was conducted on thirty patients with hand foot syndrome. Their ages ranged from 35-60 years. Patients were randomly assigned into two equal groups: Group(A) composed of 15 patients who received laser therapy 3times/week for 4 weeks in addition to their medical treatment (topical emollients) and group (B) composed of 15 patients who received only their medical treatment (topical emollients). Visual analogue scale (VAS) and hand foot syndrome-14 questionnaire were assessing both groups prior to and after 4 weeks of laser therapy using scanner laser.

The results of this study revealed significant decrease of VAS and hand foot syndrome-14 questionnaire in the study group (group A) that agreement with Latifyan et al. ${ }^{(9)}$, Zecha et al. ${ }^{(10)}$ and Avci et al. ${ }^{11)}$. Latifyan et al. ${ }^{\left({ }^{(9)}\right.}$ tested the efficacy of low-level laser therapy in reducing hand foot syndrome. A convenience sample of 31 patients with neoplastic diseases received cancer therapy included capecitabine in 16 (51\%) patients; liposomal doxorubicin in 5 patients, sunitinib in 4 patients and cytosine arabinoside, sorafenib, regorafenib, paclitaxel, docetaxel, and R-CHOP each in one patient that was recruited from Oncology Units of a General Hospital. Each patient served as his/her own control in the sense that only one hand or one foot was treated with PBM and the other received a sham irradiation. The treated side (right or left) was determined at random, using block randomization. Only the observer responsible for administering the PBM treatment was aware of the result of the randomization. The other observer remained blinded throughout the study. The patient was unaware of the side effectively treated as he/she was physically blinded during the procedure. The PBM treatment was given using a Mobile Thor Laser with a 69-diode type led probe $(34 \times 660 \mathrm{~nm}$ LED). The energy delivered was $2 \mathrm{~J} / \mathrm{cm}^{2}$ with an intensity of $500 \mathrm{mU}$. PBM or sham therapy was applied to the whole surface of the palms and soles, 3 times a week. Emollients could be used according to the patient's preferences. The patients were evaluated, by the blinded observer, after 2 weeks of therapy. The patients were asked to evaluate local pain using an analogic scale from 0 to 10 and record it weekly. In the same time, the patient expressed his/her overall satisfaction over the treatment, on a special form, weekly. We found a significant benefit from PBM as compared to sham treatment ( $\mathrm{p}=0.03$ using McNemar test) and a decrease of pain in $49 \%$ of the patients. No adverse reactions were observed. The study findings support the efficacy of low-level laser therapy in treatment of hand foot syndrome.

Zecha et al. ${ }^{(10)}$ studied a task force consisting of an international multidisciplinary panel of clinicians and researchers with expertise in the area of supportive care in cancer and/or PBM clinical application and dosimetry was formed. The mission of this group was to aid in the design of PBM study protocols, identify validated outcome measures, and test the efficacy and safety of PBM for the management of complications related to cancer therapy. They found that PBM have potential applications in the management of a broad range of side effects of (chemo) radiation therapy (CRT) in patients being treated for HNC. For OM management, optimal PBM parameters identified were as follows: wavelength, typically between 633 and $685 \mathrm{~nm}$ or $780-830 \mathrm{~nm}$. Energy density was laser or light-emitting diode (LED) output between 10 and $150 \mathrm{~mW}$, dose of $2-3 \mathrm{~J}\left(\mathrm{~J} / \mathrm{cm}^{2}\right)$, and no more than 
$6 \mathrm{~J} / \mathrm{cm}^{2}$ on the tissue surface treated for two to three times a week up to daily. Emission type, pulsed $(<100 \mathrm{~Hz})$ and route of delivery, intraorally and/or trans cutaneous. To facilitate further studies, we propose potentially effective PBM parameters for prophylactic and therapeutic use in supportive care for dermatitis, dysphagia, dry mouth, dysgeusia, trismus, necrosis, lymphedema, speech alterations and hand foot syndrome

Avci et al. ${ }^{(11)}$ found that Low-level laser therapy (LLLT) is a fast-growing technology used to treat a multitude of conditions that require stimulation of healing, relief of pain and inflammation, and restoration of function. Although the skin is the organ that is naturally exposed to light more than any other organ, it still responds well to red and near-infrared wavelengths. The photons are absorbed by mitochondrial chromophores in skin cells. Consequently, electron transport, adenosine triphosphate (ATP) nitric oxide release, blood flow, reactive oxygen species increase and diverse signaling pathways are activated. Stem cells can be activated allowing increased tissue repair and healing. In dermatology, LLLT has beneficial effects on wrinkles, acne scars, hypertrophic scars, and healing of burns. LLLT can reduce UV damage both as a treatment and as a prophylaxis. In pigmentary disorders such as vitiligo, LLLT can increase pigmentation by stimulating melanocyte proliferation and reduce depigmentation by inhibiting autoimmunity. Inflammatory diseases such as psoriasis and acne can also benefit.

Akerzoul and Chbicheb ${ }^{(12)}$ found that Laser therapy has beneficial therapeutic effects, such as analgesia, anti-inflammation and wound healing. Although not completely known, laser therapy acts through different suggested mechanisms including modulation of natural substances (histamine, acetylcholine, opioid peptides, kinins, prostaglandin, interleukin, interferon and tumor necrosis factor), changes in impulse conduction of nociceptors and effects on lymphocyte metabolism. Laser therapy can also improve microcirculation and oxygenation of the tissue and stimulate epithelial, endothelial and mesenchymal cell growth. Laser treatment of recurrent aphthous stomatitis is an easy, fast and pain-free procedure. The study have shown that ulcers treated by laser therapy provide immediate pain relief and fewer recurrences in the future. The main advantage of the LLLT compared to other treatment options is that it can be used for all the causes of the disease both without having any side effects and without the risk of medication overdose.

\section{CONCLUSION}

Based on this study, it could be concluded that PBM might represent a useful approach for the management of HFS.

\section{REFERENCES}

1. Lalabonova M, Rakaraddi M, Pai J et al. (2016): The use of diode laser versus 5\% amlexanox: a comparison of treatment effects in a cohort of patients with minor aphthous ulcers. Oral Surgery, Oral Medicine, Oral Pathology and Oral Radiology, 121 (3): 269-273.

2. Degen A, Alter M, Schenck F et al. (2010): The handfoot syndrome associated with medical tumor therapy - classification and management. J Dtsch Dermatol Ges., 8: 652-61.

3. Lopes N, Plapler H, Chavantes M et al. (2009): Cyclooxygenase-2 and vascular endothelial growth factor expression in 5-fluorouracil-induced oral mucositis in hamsters: evaluation of two low-intensity laser protocols. Support Care Cancer, 17: 1409-1415.

4. Mangili G, Petrone M, Gentile C et al. (2008): Prevention strategies in palmar-plantar erythrodysesthesia onset: the role of regional cooling. Gynecol Oncol., 108: 332-335.

5. Chung H, Dai T, Sharma S et al. (2012): The nuts and bolts of low-level laser (light) therapy. Ann Biomed Eng., 40 (2): 516-533.

6. Aggarwal H, Pal Singh M, Nahar $P$ et al. (2014): Efficacy of low-level laser therapy in treatment of recurrent aphthous ulcers-a sham controlled, split mouth follow up study. Journal of Clinical and Diagnostic Research, 8 (2): 218-221.

7. Gould D (2001): Visual Analogue Scale (VAS). Journal of Clinical Nursing, 10: 697-706.

8. Clark A, Vahdat L (2004): Chemotherapy-induced palmar-plantar erythrodysesthesia syndrome: Etiology and emerging therapies. Support Cancer Ther., 1: 213218.

9. Latifyan S, Genot M, Fernez B et al. (2020): Use of low-level laser therapy (LLLT) or photobiomodulation (PBM) for the management of the hand-foot syndrome (HSF) or palmo-plantar erythrodysesthesia (PPED) associated with cancer therapy. Support Care Cancer, 28 (7): 3287-3290.

10. Zecha J, Raber-Durlacher J, Nair $R$ et al. (2016): Low-level laser therapy/photobiomodulation in the management of side effects of chemoradiation therapy in head and neck cancer: part 2: proposed applications and treatment protocols. Support Care Cancer, 24 (6): 2793-2805.

11. Avci P, Gupta A, Sadasivam M et al. (2013): Lowlevel laser (light) therapy (LLLT) in skin: stimulating, healing, restoring. Semin Cutan Med Surg., 32 (1): 41 52.

12. Akerzoul N, Chbicheb S (2018): Low laser therapy as an effective treatment of recurrent aphtous ulcers: a clinical case reporting two locations. Pan Afr Med J., 30: 205-209. 